\title{
Emile Gabel - und die Zukunft der UCIP
}

Man konnte ihn nicht einmal mehr identifizieren. Bloß eine Mappe mit einigen Dokumenten vom letzten Tag in Bogota verwies auf P. Emile Gabel A. A., einen der 63 Toten der in der Nacht zu Mittwoch, den 6. März 1968, zerschellten und ausgebrannten Air-France-Boeing 707 auf der Antilleninsel Guadelupe.

Mit Entsetzen vernahm die katholische Presse in der ganzen Welt die Todesnachricht. Man war so sehr an ihn gewöhnt, daß der Gedanke gar nicht aufkommen konnte, was wäre, wenn einmal...

P. Gabel war Generalsekretär der Katholischen Weltunion der Presse (Union Catholique Internationale de la Presse: UCIP). Er war es zwölf Jahre lang mit ganzer Kraft. Er setzte seine Gesundheit ebenso ein wie das Geld, das er verdienen mußte, um das Sekretariat selbst finanzieren zu können! Der deutschsprechende Elsässer, am 1. September 1908 in Drusenheim geboren, stand immer im Dienste der Publizistik. Schon während des Krieges, nachdem er vorher einige Jahre in seinem Assumptionistenorden Dogmatik gelehrt hatte, übernahm er den Buchverlag im Maison de la Bonne Presse. In der Bonne Presse erschien auch die Tageszeitung „La Croix“, in der er von 1949 bis 1957 als Chefredakteur praktizierte, was er von 1957 an

„Für Ihr Heft 3 bin ich natürlich gerne bereit, einen Beitrag von ungefähr $6-7$ Schreibmaschinenseiten zu liefern, vielleicht über die Gründe, die uns zur Wahl unseres Kongreßthemas [„Welt - Kirche - Presse", für den 8. Weltkongreß der katholischen Presse, 2. 6. Juli 1968 in Berlin] bewogen haben. Teilen Sie mir bitte den Ablieferungstermin für diesen Artikel mit."

Emile Gabel am 19. Januar 1968 an CS

als Generalsekretär der Katholischen Weltunion der Presse werbend, dozierend und organisierend verfocht: das Recht auf Information in der Kirche. 1963 hatte er das Problem so klar formuliert, daß das Zitat seither immer wieder verwendet wurde:

"Spricht man vom Recht auf Information, so sollte man nicht sagen: Dieses Recht besteht sogar in der Kirche, sondern: Dieses Recht besteht um so mehr in der Kirche. Beruht nämlich das Recht auf Information auf menschlicher Solidarität und sozialer Verantwortung, so gibt es keine Gesellschaft, wo es geachteter und entwickelter sein sollte als gerade in der Kirche." 1

Er konnte sich dabei bereits auf die Enzyklika Johannes' XXIII. Pacem in terris berufen, die unter den universalen Menschenrechten das Recht auf objektive Information anerkannt hat. Dieser Gleichklang der Gedanken von Johannes XXIII. und Pater Gabel ist nicht zufällig: Als Papst Johannes XXIII. noch Nuntius Roncalli in Paris war, zählte zu seinen häufigsten Gesprächspartnern - P. Emile Gabel.

Es kam das Konzil und mit ihm die große Aufgabe, auch zu den Massenmedien Stellung zu beziehen. Leider war dieses Thema einer der ersten Punkte auf der Konzilstagesordnung, als den Konzilsvätern noch nicht der später so enge und 
lebhafte Umgang mit der Presse vertraut war. Es wäre dann ganz anders ausgefallen, deutete Kardinal Jaeger an. Angesichts der bisherigen Hilflosigkeit der Kirche gegenüber den Massenmedien konnte es darum auch einem P. Gabel nicht gelingen, dem Dekret über die Massenmedien allzuviel Zukunftsrichtung und Inhalt zu verleihen. Immerhin: Es gelang ihm, im Konzilstext das entscheidende „Recht auf Information" unterzubringen: "Es gibt in der menschlichen Gesellschaft ein Recht auf Information über alle Tatsachen, die den Menschen als einzelnen oder als Mitgliedern der Gesellschaft je nach ihrer besonderen Situation zu wissen zukommt." Eine solche Formulierung hat Folgen. Auch wenn in der Kirche immer noch der publizistische Kleinmut groß geschrieben wird: „Man darf die Wasser, die man ruhig haben will, nicht aufwühlen." So zeichnete 1967 in Wien P. Gabel die Haltung der Ängstlichen. Er zeigte aber sofort, wohin dieses Um-die-Sache-herum-Reden führt: „Aber ehe man sich versieht, sind die Wasser faul geworden!“

Aus dieser Presse-Sicht hat Gabel um der Freiheit des Wortes in der Kirche willen in München über die Funktion der Presse gesagt: „Sie wird um so wirksamer sein, je mehr die katholische Presse zu einer wesensgemäßen Eigenständigkeit und Distanz zur Hierarchie gelangt und die andere, weltliche Presse der Kirche freundlicher begegnet."

Was nützen aber die schönsten Grundsätze, wenn die einfachsten technischen Mittel für vernünftige Pressearbeit fehlen? P. Gabel wußte vor seinen Reisen, Gesprächen und Begegnungen, wie schlecht es um die katholische Presse, vor allem in den Ent-

\section{[P. Gabel und die Publizistikwissenschaft]}

In seinen letzten Lebensjahren, vor allem aufgrund der Konzilserfahrungen, bemühte sich P. Gabel intensiv um die Förderung wissenschaftlicher Forschung und L.ehre im Bereich der Kommunikationsmittel. Daß die Hochschulinstitute, die sich mit der Ausbildung von Journalisten befassen, in einer eigenen internationalen Föderation zusammengeschlossen und der UCIP eingegliedert wurden, geht entscheidend auf seine Initiative zurück. Als Fernziel schwebte ihm ein internationales Forschungsinstitut im Rahmen der Pariser Katholischen Universität vor, das durch seine Arbeit jene Lücken schließen sollte, die beim Zustandekommen des Konzilsdekrets über die Kommunikationsmittel und danach bei den Bemühungen um eine Pastoralinstruktion so stark fühlbar geworden sind. Ein großer Teil seiner Aktivität im Hintergrund galt der Vorbereitung dieses großen Vorhabens, den Kontakten mit Zeitungs- und Publizistikwissenschaftlern in Pamplona, Loewen. Nimwegen, München und anderswo, dem Suchen nach einer gemeinsamen Basis für eine unabhängige, von Tagesfragen angeregte, aber nicht bedrängte Studien- und Forschungsarbeit auf lange Sicht. Dabei beschäftigte ihn das Problem der Informations- und Meinungsfreiheit in Staat und Kirche ebenso wie die Sorge um eine Standesethik des Journalisten.

Otto B. Roegele

wicklungsländern, stand. So wurde in der letzten Zeit immer stärker die Sorge um die Presse in den Entwicklungsländern zum Schwerpunkt seiner Arbeit: Dank seiner überzeugenden Argumente entschlossen sich die deutschen Bischöfe schon 1964, durch die Aktion ADVENIAT den katholischen Massenmedien in Lateinamerika zu helfen. P. Gabel wurde Konsulent von ADVENIAT. - 1966 wurde innerhalb der UCIP eine eigene Entwicklungskommission gegründet, die sich vor allem der Kooperation und Koordination aller Hilfsmaßnahmen wie auch der Erschließung von Finanzierungsquellen widmen sollte. 
Aus dieser flüssiger werdenden Aufbauarbeit riß nun der Tod P. Gabel, - den Mann, der immer und überall da war, wo man ihn brauchte, den zähen Prediger und Vermittler in Sachen Presse. Was wird jetzt weiter? Vor allem - was wird aus der UCIP?

Wenn irgendwo das Wort des Bamberger Katholikentags, Organisationen müßten „personal erfüllt" sein, zutrifft, dann war es hier der Fall. Die UCIP war Pater Gabel. Gewiß trafen sich bei den Weltkongressen und sonstigen Tagungen Verleger, Journalisten aus allen Teilen der Welt. Aber Pater Gabel war die Seele des ganzen Unternehmens. Soll es als Omen für den Zustand der katholischen Presse in der Welt gewertet werden, daß sie nicht einmal imstande war, ihr eigenes Sekretariat zu finanzieren?

Wenn die UCIP nicht zu einer leeren Organisationshülse werden soll, muß sie ehrliche Selbstkritik üben. Enge Freunde und Mitarbeiter von Pater Gabel wissen, wie sehr ihn das Ubermaß an Problemen gegenüber den wenigen Tatsachen der Leistung bedrückt hat. Es wird nicht einfach sein, einen Nachfolger zu finden, der das Vertrauen der Kurie ebenso besitzt wie das der nationalen Bischofskonferenzen, der aufgrund seiner Fachkenntnisse von Verlegern ebenso geschätzt wird wie von Journalisten, der unvermeidliche Spannungen $\mathrm{zu}$ entschärfen wie für neue Initiativen Mitarbeiter zu gewinnen vermag.

Der Weg, den die Katholische Weltunion der Presse gehen muß, sollte nicht ohne engen, auch arbeitstechnischen Kontakt $\mathrm{zu}$ den anderen katholischen Weltorganisationen des Funks und des Films sein. Man darf nicht übersehen, daß die Welt schon heute eine Einheit bildet. Aktionen der Presse können nicht mehr ohne Abstimmung etwa mit den Plänen der Rundfunkleute durchgeführt werden. Koordination und Kooperation werden für jede Weltbewegung zur Selbstverständlichkeit. Sollte nicht die UCIP diese Perspektiven beruicksichtigen, vielleicht sogar durch eine Verlegung des Generalsekretariats nach Brüssel, wo nicht nur die meisten Diplomaten in einer europäischen Stadt versammelt sind, sondern auch das Office Catholique International du Cinéma (OCIC) seinen Sitz hat und seit kurzem die Koordinationszentrale aller europäischen katholischen Hilfswerke wie auch das soziologische Institut Pro Mundi Vita lokalisiert ist?

Emile Gabel hatte die Hauptlast der Verantwortung für das Wohl und Wehe der katholischen Presse zu tragen. Wenige wissen, wie schwer er manchmal gerungen hat, um nicht zu resignieren. Wir haben es uns zuweilen zu leicht gemacht, wenn wir - um zu einem Konferenzende zu kommen - uns trösteten: P. Gabel wird es schon machen. Das hat er wahrhaft getan. Jetzt stehen wir allein - ohne ihn vor den Problemen. "Wir können nur miteinander versuchen, in seinem Geiste die Arbeit fortzusetzen", heißt es im Beileidsbrief eines Freundes von P. Gabel.

Otto Kaspar, Vorsitzender der Entwicklungskommission der UCIP

Anmerkungen:

1. Emile Gabel: Recht auf Information. Das Konzil und die Mittel der Massenkommunikation, in: "Wort und Wahrheit", Freiburg/Br. 18: 1963, S. 409_416, hier S. 415.

2. Dekret "Inter mirifica" (vom 4. 12. 1963) des II. Vaticanums, Nr. 5. 


\section{Alfons Nowak $\uparrow$}

Als vor 20 Jahren eine Handvoll Journalisten, Verleger, Autoren und Funkpublizisten im deutschen Katholizismus Umschau hielten, was Krieg und Nachkrieg, Hitler- und Besatzungszeit übriggelassen hatten, war man bald der einhelligen Meinung, es lohne sich wieder, auf dem Boden neugewonnener Pressefreiheit katholische Publizistik zu schaffen. Um den Jesuitenpater Dr. Jansen Cron, vielen der Übriggebliebenen als mutiger Pressepater der Jugendbewegung Neudeutschland noch in guter Erinnerung, versammelten sie sich als „Gesellschaft Katholischer Publizisten Deutschlands e. V.“. Nicht nur Presseleute, sondern von Anfang an auch Funk- und Fernsehpublizisten, Zeitungswissenschaftler und Buchautoren waren die Gründungsmitglieder.

In jenen Tagen, als „Organisieren" bloß ein Deckwort war für das Besorgen notwendiger Lebensmittel und Kleidungsstücke am Rande der Legalität, lief der Gesellschaft ein Mann über den Weg, der von Organisation mehr verstand als die meisten anderen. Es war der in Flatow bei Schneidemühl am 8. Oktober 1911 geborene Alfons Nowak, der keine Gelegenhejt ausließ, in seiner Eigenschaft als Geschäftsführer der Diözesan-Katholikenausschüsse des Erzbistums Köln durch einfallsreiches Organisationstalent zu imponieren. Der Appell der Publizisten an seinen „jugendbewegten "Idealismus war nicht vergeblich. Alfons Nowak wurde ihr erster Geschäftsführer, ein beharrlich-sicherer und getreuer Verwalter des komplexen Gebildes einer Gesellschaft von Individualisten.

Die Vorbereitung und der reibungslose Ablauf vieler Tagungen im In- und Ausland ist nur seiner Findigkeit, seinem in aufregenden Situationen beruhigenden Temperament zu verdanken. Die Katholisch-Evangelischen Begegnungen fanden in ihm, dem Diaspora-Katholiken aus dem verlorenen deutschen Osten, einen anteilnehmenden Organisator. Einfallsreich wußte er auch die Kontakte mit ausländischen Kollegen, vor allem auf den von ihm gleichfalls vorbereiteten Deutsch-Französischen Treffen und auf den Tagungen der Publizisten des deutschen Sprachraumes, zu pflegen.

Daß diese positive Darstellung seiner publizistischen Verdienste an dieser Stelle überhaupt möglich war, ist nur einem todtraurigen Anlaß zuzuschreiben. Seine sympathische Bescheidenheit hätte den Freunden jedes ihm selbst zugedachte Lob verbeten: Alfons Nowak starb am 14. Februar 1968 im Alter von 56 Jahren nach kurzem Krankenhausaufenthalt, der ihm Heilung von einem lange verborgen gehaltenen Leiden bringen sollte. Betrauert von seiner Frau und seinen vier Kindern, von seiner ihm zu Dank verpflichteten Gesellschaft Katholischer Publizisten und der Gemeinde Lövenich, deren langähriger verdienstvoller Bürgermeister gewesen war. Briefe aus vielen Staaten, aus allen Bereichen der Publizistik geben die Bestürzung über den plötzlichen Tod dieses kontaktreichen Mannes wieder, der zu den führenden Persönlichkeiten katholischer Publizistik gehörte. Was andere mit Feder, Maschine und Tonband darstellen: Alfons Nowak schrieb es mit dem Herzen.

Konrad Simons,

Vorsitzender der Gesellschaft

Katbolischer Publizisten Deutschlands e. V. 


\section{Rochus Spiecker $†$}

Am 20. Februar 1968 ist nach längerer Krankheit Dr. theol. Rochus Spiecker, Kölner Dominikanerpater, in Bensberg gestorben; er war einer der fähigsten praktischen Publizisten des katholischen Deutschland. Der am 24. Juli 1921 in Berlin geborene Wolf Rochus Spiecker absolvierte nach Kriegsteilnahme und Gefangenschaft seine Studien bei den Dominikanern in Walberberg, nachdem er bereits 1939 in den Prediger-Orden eingetreten war. 1949 wurde er zum Priester geweiht, - bis 1952 konnte er in Rom weiterstudieren, wo er Lizentiat, Lektorat und Doktorat erwarb. Nach Deutschland zurückgekehrt (1953-1960 Bundeskaplan der Deutschen Pfadfinderschaft St. Georg) entfaltete er als Prediger, Funk- und Pressemitarbeiter vielfältige publizistische Tätigkeit, die durch zwei Qualitäten ausgezeichnet war: Humor und Unbefangenheit, - Unbefangenheit besonders in der Bevorzugung neutraler oder auch schon ausgeprägt liberaler Medien. Einiges Aufsehen erregte seine Mitarbeit am liberalen (und auch damals schon einem extremen Liberalismus sich zuneigenden) Hamburger Wochenblatt "Die Zeit“. Der Rundfunk schätzte seinen präzisierenden Humor („Den Kern freilegen, ohne die Haut zu verletzen“), Werner Höfer nannte ihn einen „Kreuzfahrer für das Vernünftige und Ketzer gegen das Törichte". Rochus Spiecker selbst, der 1962 den Aachener Karnevals-,,Orden wider den tierischen Ernst" entgegennahm, antwortet spielerisch: „Wie ich zum Schreiben kam? Wie ich an den Funk, das Fernsehen, den Film geriet? Jedenfalls nicht aus ,Taktik'. Mich fesselt das Spiel, das mehr ist als ,Spiel'. Das Theater, das mehr ist als ,Theater'. Mich fasziniert der Versuch, das Leben zu deuten, indem man es verdichtet und überschaubar macht, ohne es zu abstrahieren." Als publizistische Potenz, aber auch als Entkrampfungstherapeut katholischer Publizistik wird Spiecker schwer zu ersetzen sein.

M. S.

\section{Kurt Baschwitz zum Gedächtnis}

Mit Recht darf man in der Wissenschaft von der Publizistik von einem verlustreichen Wintersemester 1967/68 sprechen. Ende November 1967 starb in Löwen Professor DeVolder (58), - am 6. Januar 1968 (vgl. CS 1:1, S. 25 und S. 38) folgte ihm der Amsterdamer Publizistik-Wissenschaftler Professor Dr. Kurt Baschwitz (81).

Obwohl Baschwitz schon seit Jahren emeritiert war, hat er sich eigentlich nie wirklich zur Ruhe gesetzt; er wurde sozusagen am Schreibtisch plötzlich abberufen. Noch vor wenigen Jahren (1963) veröffentlichte er sein Meisterwerk „Hexen und Hexenprozesse". Die Problematik des Massenwahns hat ihn schon früh gefesselt; noch in seiner Zeit in Deutschland (vor 1933) erschien seine erste Veröffentlichung darüber: "Der Massenwahn" (1. Aufl. 1923; 3. Aufl. 1932). Bevor amerikanische Untersuchungen zur Strukturierung des fast ungreifbaren Begriffes „Masse“ neue Wege zur Erkenntnis anbahnten, hat Baschwitz in historisch belegten Massenaktionen die Gruppe hinter der „Masse" erkannt: in seiner Unterscheidung der gewalttätigen Minderheiten und der duldsamen Mehrheiten. Wie haben uns s. Z. seine Darlegungen über die Schreckensherrschaft der Schlechtesten und die stumme Panik gefesselt; 
wie hat uns die Feststellung begeistert, daß der einzelne mit Zivilcourage oft im Stande ist, einen Massenwahn zu durchbrechen.

Auch mit seinem Grundsatz, daß die Leser das Wichtigste an einer Zeitung sind, war dieser erste niederländische Presse-Professor manchem publizistozentrischen Zeitungswissenschaftler im funktionalen Denken weit voraus. - Die umfangreiche Emeritatsarbeit an „Hexen und Hexenprozesse“, die er allein, ohne Stützung im Rahmen eines wissenschaftlichen Instituts, schuf, hatte ihn, dem Anschein nach, völlig erschöpft. Dank der guten Pflege seiner Frau und Mitarbeiterin Ilse Baschwitz ist er dann doch wieder gesund geworden. Bei meinen letzten Besuchen in Amsterdams Prinsengracht 644 war er schon wieder erfüllt von den Plänen für eine neue Gestaltung seiner "Massenpsychologie".

Sein Interesse blieb bis zuletzt dem publizistischen Fach zugewandt. Als ich ihn zum letzten Mal besuchte, wollte er genau über die Lage des Faches an den deutschen Hochschulen informiert werden, besonders auch darüber, was ich als Niederländer auf dem Lehrstuhl Walter Hagemanns an der deutschen Universität Münster für Erfahrungen gemacht hatte und was für Arbeiten in Münster unter meiner Leitung im Entstehen begriffen seien; er fragte mit dem gleichen Interesse, mit dem er einst meiner ersten religionspublizistischen Studie "Kerkgang om Nieuws" ein Vorwort auf den Weg gegeben hatte. Obwohl Baschwitz seine neue niederländische Heimat als das Land seiner Freiheit nach der Flucht 1933 aus Berlin lieb gewonnen hatte, sprach er noch immer vorzugsweise deutsch. Als ich ihm zu seinem 80 . Geburtstag bei dem aus diesem Anlaß veranstalteten Empfang in Amsterdam in der niederländischen Sprache im Namen der Deutschen Gesellschaft für Publizistik- und Zeitungswissenschaft huldigte, antwortete er deutsch. Die ihm angebotene Ehrenmitgliedschaft dieser Gesellschaft verstand er so, daß er auch bier noch dazugehörte. Nazismus und Deutschland waren für ihn nicht kongruent. Er pflegte eine wahrlich europäische Bipatridie.

Bei der Einäscherung in Velsen führte sein Amsterdamer Nachfolger, Professor Maarten Rooij, für uns alle das Wort: Ehre bleibe dem Gedächtnis des großen Gelehrten und humanen Menschen Sigfried Kurt Baschwitz.

Henk Prakke 